\title{
Magnetic Resonance Imaging Diagnosis of Hepatocellular Carcinoma in Cirrhotic Patients: Where Are We Now?
}

\section{Diagnóstico de Carcinoma Hepatocelular por Ressonância Magnética em Doentes Cirróticos: Onde nos Encontramos?}

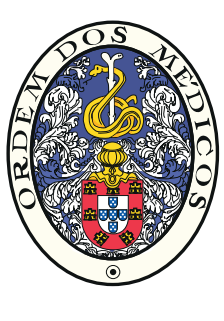

\author{
Miguel RAMALHO $\bigotimes^{1,2}$, Mamdoh ALOBAIDY ${ }^{1,3}$, António P. MATOS ${ }^{1,2}$, Richard C. SEMELKA ${ }^{1}$ \\ Acta Med Port 2016 May;29(5):293-296 - http://dx.doi.org/10.20344/amp.6665
}

Keywords: Carcinoma, Hepatocellular/diagnosis; Liver Cirrhosis/diagnosis; Liver Neoplasms/diagnosis; Magnetic Resonance Imaging. Palavras-chave: Carcinoma Hepatocelular/diagnóstico; Cirrose Hepática/diagnóstico; Neoplasias do Fígado/diagnóstico; Ressonância Magnética.

\section{INTRODUCTION}

Hepatocellular carcinoma (HCC) is the sixth most commonly diagnosed cancer worldwide, usually developing within a cirrhotic background.

The development of HCC in a cirrhotic liver is described either as de novo hepatocarcinogenesis or as a multistep progression, from low-grade (LGDNs) to high-grade dysplastic nodules (HGDNs), then some HGDNs develop microscopic foci of HCC, progress to small HCC, and finally to invasive carcinoma.

$\mathrm{HCC}$ is very aggressive and is usually associated with poor prognosis and outcome. Nonetheless, patients diagnosed at early stages are eligible for potentially curative therapies including surgical resection, liver transplantation, or locoregional treatment, such as radiofrequency or microwave ablation. In this population, stage-driven treatment results in 5-year survival rates in the range of $50-70 \% .{ }^{1}$ Therefore, diagnosing $\mathrm{HCC}$ at early stages is critical.

\section{Surveillance for HCC}

Surveillance for HCC is a controversial topic. Currently, gray-scale ultrasound (US) is widely used for the surveillance of HCCs in high-risk individuals and has been the modality most commonly used for screening for HCC. A recently updated practice guideline for the management of HCC by the American Association for the Study of Liver Diseases (AASLD) recommended that surveillance of $\mathrm{HCC}$ should be based on US at 6 -month intervals. ${ }^{2}$

The reason for US being the most common initial imaging test used for screening and surveillance of HCC is primarily due to the ease of access, absence of risks, noninvasiveness, good acceptance by patients and relative lower initial per study cost compared with with computed tomography (CT) or magnetic resonance imaging (MRI). Reported sensitivity and specificity are quite variable but is highly dependent on the expertise of the operator and patient's morphotype. Previous studies have shown a signifi- cant lower detection rate of $\mathrm{HCC}$ compared to multidetector $\mathrm{CT}$ and MRI. ${ }^{3}$ Furthermore, the technique is poor to detect small HCCs.

According to the updated AASLD and European Association for the Study of the Liver (EASL) guidelines, the diagnostic algorithm of HCC starts from suspected nodules found on US surveillance. When a subcentimeter nodule is detected by US, it should be followed by US every 3 months until the nodule is no longer visualized, remains stable for 18 to 24 months, or grows larger than $10 \mathrm{~mm}$ in size. However, in clinical practice, a dedicated MRI or CT is recommend once a nodule is detected at US, even if it is smaller than $10 \mathrm{~mm}$. To date, confirmation of HCC is based on the hemodynamic feature of the nodules, i.e., enhancement on the arterial phase and washout on portal or equilibrium phase.

The standard CT protocol comprises a multiphase dynamic imaging, which consists of acquisition of unenhanced images followed by acquisition of arterial, portal venous and/ or equilibrium phase images after iodinated contrast material administration. Nevertheless, CT comes at a cost in the form of radiation exposure, especially if used for follow-up purposes. Additionally, a subset of patients with hepatorenal disease may be at increased risk for worsening renal function from iodinated contrast agents.

MR imaging offers advantages over $\mathrm{CT}$, including lack of ionizing radiation, higher contrast resolution, higher safety profile of contrast agents, ability to use both extracellular and hepatocyte-specific contrast media, characterization of occasional problematic pseudo-lesions on US or CT such as focal fatty infiltration or focal fatty sparing, and overall better accuracy in the detection and characterization of focal lesions. ${ }^{4}$

Several studies have demonstrated an increased sensitivity and specificity of contrast enhanced MRI (DCE$\mathrm{MRI}$ ) over dynamic CT for the detection and character-

1. Department of Radiology. University of North Carolina. Chapel Hill. United States of America

2. Department of Radiology. Hospital Garcia de Orta. Almada. Portugal.

3. Department of Radiology. King Faisal Specialist Hospital and Research Center. Riyadh. Saudi Arabia.

$\square$ Autor correspondente: Miguel Ramalho. jmpmramalho@gmail.com

Recebido: 01 de junho de 2015 - Aceite: 26 de outubro de 2015 | Copyright @ Ordem dos Médicos 2016 
ization of $\mathrm{HCC}$ of all sizes. ${ }^{5}$ Many MR imaging features deserve attention, however enhancement is still considered the most important and reflect sequential changes in the intra-nodular blood supply during hepatocarcinogenesis. As cellular atypia progresses toward malignancy, blood supply becomes more arterialized. Arterial hyper-enhancement
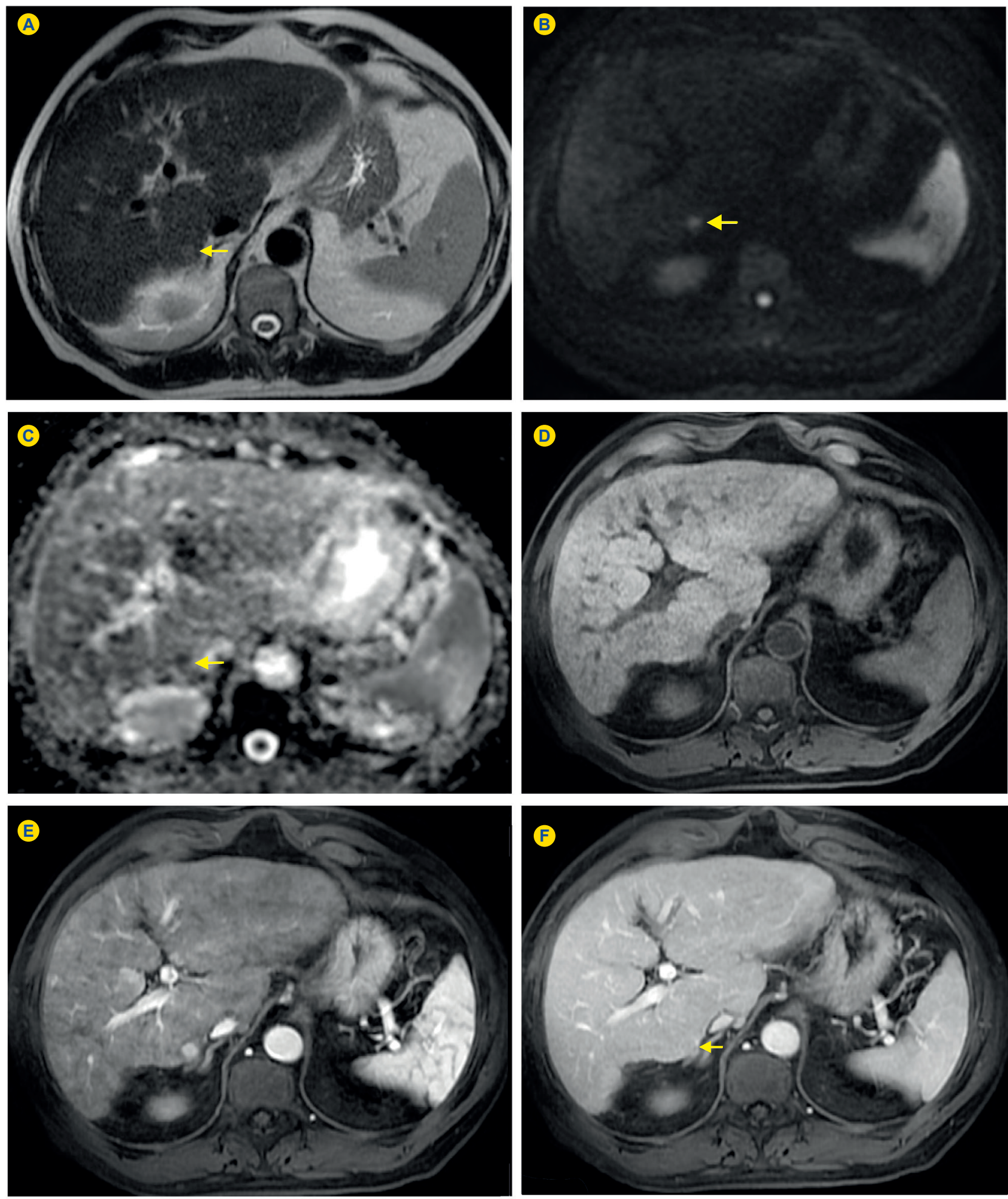

Figure 1 - Small HCC in a patient with alcoholic chronic liver disease. Axial SS-FSE T2-WI (A), axial DWI $\left(B=600 \mathrm{sec} / \mathrm{mm}^{2}\right)$ and corresponding apparent diffusion coefficient (ADC) map (C), axial pre- (D) and postcontrast fat-suppressed 3D-GRE T1-WI in the arterial (E) and interstitial $(F)$ phases. A small $10 \mathrm{~mm}$ nodule is depicted on the right hepatic lobe with minimal high signal intensity on T2-WI (arrow, A). The nodule shows hyperintensity on DWI (arrow, B) and low signal on the corresponding ADC map (arrow, C), consistent with restriction to diffusion. On DCE-MRI, the nodule shows arterial hyperenhancement, with subtle washout on late phases (arrow, F). This example illustrates the contribution of DWI to improve the sensitivity for the diagnosis of HCC 
is the single most critical imaging feature of $\mathrm{HCC} ; 6$ however, it can be also seen in HGDNs and arterio-portal shunts. The diagnosis of HCC on the basis of the typical enhancement criteria of arterial hyper-enhancement and venous washout is highly sensitive and specific particularly for tumors greater than $20 \mathrm{~mm} .^{6,7}$ The sensitivity decreases for HCCs smaller than $20 \mathrm{~mm}$ as small HCCs tend to be hypervascular but not show washout on delayed images. ${ }^{8,9}$ In one series of $60 \mathrm{HCCs}$, smaller than $20 \mathrm{~mm}, 85 \%$ of these lesions were hypervascular, and only $61.7 \%$ of which showed washout. ${ }^{8}$

In addition to wash-in/washout features, several ancillary signs only depicted with MRI have been described, including intratumoral lipid, high T2 signal or hyperintensity on high b value diffusion-weighted imaging (DWI), increasing the specificity for HCC.

Intratumoral lipid is relatively uncommon (sensitivity for HCC of $12-37 \%),{ }^{6,10}$ and is more frequently present in the form of intracellular lipid, which may be identified with chemical shift imaging. As a rule, any lipid-containing tumor in a cirrhotic liver should be viewed with suspicion, especially when the lesion diameter is $>15 \mathrm{~mm} .{ }^{10}$

The appearance of HCC on T2-weighted images is variable. HCCs tend to show minimal to mildly increased signal intensity on T2-weighted images when large. One study reported increased specificity in hypervascular nodules when combined with increased T2 signal up to $95 \% .{ }^{6}$ Recent studies have shown that the addition of T2 weighted imaging to gadolinium-enhanced MR imaging improves the diagnostic performance in the detection of HCC compared to isolated DCE-MRI. This is particularly true for HCCs smaller than 10 or $20 \mathrm{~mm}$, which may show hypervascularity, but might not show washout, discriminating them from HGDNs. ${ }^{11}$

Diffusion weighted imaging (DWI) is a form of MR imaging based upon measuring the random displacement of water molecules within a voxel of tissue, known as Brownian motion. This movement of intracellular water molecules is restricted by the presence of cell membranes. In highly cellular tissues, such as neoplasms, diffusion is restricted due to the relative larger intracellular volume and high density of cellular membranes. DWI exploits this phenomenon and its image contrast is based on differences in the mobility of water protons (as a measure of cellularity), between different tissues. Several studies have shown encouraging results with DWI, suggesting a good diagnostic performance in the detection of HCC in patients with chronic liver disease, and equivalent to conventional contrast-enhanced for lesions greater than $20 \mathrm{~mm}$ in size. ${ }^{12}$ The greatest benefit relies on the combined use of DWI with conventional DCE-MRI (Fig.1). A recent meta-analysis found that DWI combined with conventional DCE-MRI performed significantly better than either DWI alone or conventional DCE-MRI alone (pooled sensitivity and specificity: $93 \%$ and $84 \%$ combined, $81 \%$ and $89 \%$ DWI, $79 \%$ and $62 \%$ DCE). Therefore, an additional acquisition of DWI is being implemented in many abdominal protocols. ${ }^{12}$

Intravenous MR contrast agents can be divided into extracellular (ECA) and hepatobiliary contrast agents
(HCA). ECA equilibrate with the extracellular fluid space after intravenous injection and are excreted by glomerular filtration. As ECA, HCA allow multi-phase dynamic post-contrast imaging. Furthermore, they show some degree of biliary excretion, permitting a late hepatobiliary phase acquisition. Due to the action of known cellular membrane transporters, only normal functioning hepatocytes take up HSA and excrete them to the biliary tree Hepatobiliary phase images are easy to recognize because both the liver and the bile ducts are markedly enhanced. The blood vessels as well as all non-hepatocellular lesions and lesions with impaired hepatocytes appear hypointense. HCA are modifying the MR diagnosis of HCC. Two HCAs are currently available: gadoxetate disodium (Eovist ${ }^{\circledR} / \mathrm{Pri}-$ movist ${ }^{\circledR}$; Bayer Healthcare) and gadobenate dimeglumine (MultiHance ${ }^{\circledR}$; Bracco Diagnostics), which combine extracellular properties with liver-specific properties, allowing both DCE-MRI and hepatobiliary imaging. Gadoxetic acid is more highly liver-specific with an uptake of approximately $50 \%$ of the injected dose, allowing hepatobiliary imaging within 20 min from the time of injection, compared with an uptake of $35 \%$ for gadobenate dimeglumine, which allows for hepatobiliary imaging within 2-3 hours. The combination of routine DCE-MRI and hepatobiliary imaging has been reported to be both sensitive and specific for HCC. A recent meta-analysis found a pooled sensitivity of $91 \%$ and specificity of $93 \% .^{13}$

There are several major up-to-date imaging-based classification systems for HCC worldwide including three in the United States [the AASLD, the Liver Imaging Reporting and Data System (LIRADS) and the Organ Procurement and Transplantation Network/United Network for Organ Sharing (OPTN/UNOS) system], two in Asia [the Japanese Society of Hepatology (JSH) system and the Asian Pacific Association for the Study of the Liver (APASL) system], and one in Europe (EASL). All these classification systems still rely on dynamic enhancement features of the liver mass (i.e., washin and washout) for the establishment of final diagnosis of HCC. To date, only LIRADS has started using ancillary features. For example, in the absence of typical enhancement features, ancillary features suspicious for malignancy such as hyperintense signal on T2W imaging, can be used to upgrade a mass to LR-4 (probably HCC), but not yet to LR-5 (definitely HCC). On the other hand, ancillary features that favor benignity can also be used to downgrade the LR category. ${ }^{14}$

In summary, MR imaging is currently the reference standard for the noninvasive evaluation of the cirrhotic liver. The combination of arterial phase hyper-enhancement and delayed washout allows a definitive diagnosis of HCC, eliminating the requirement for confirmatory biopsy. However, the sensitivity of these hallmarks is relatively low for small HCCs. Ancillary MR imaging features assessed by conventional and novel practices, as well as the addition of HCAs seem to improve the sensitivity for early HCC detection, which may ultimately alter clinical management and potentially improving patient's survival. 


\section{CONFLICTS OF INTEREST}

The authors have no conflicts of interest to declare.

\section{FINANCING SUPPORT}

This work has not received any contribution, grant or scholarship.

Diagnosis of hepatic nodules $20 \mathrm{~mm}$ or smaller in cirrhosis: Prospective validation of the noninvasive diagnostic criteria for hepatocellular carcinoma. Hepatology. 2008;47:97-104.

9. Watanabe A, Ramalho M, AIObaidy M, Kim HJ, Velloni FG, Semelka RC. Magnetic resonance imaging of the cirrhotic liver: An update. World J Hepatol. 2015;7:468-87.

10. Yu JS, Chung JJ, Kim JH, Kim KW. Fat-containing nodules in the cirrhotic liver: chemical shift MRI features and clinical implications. AJR Am J Roentgenol. 2007;188:1009-16.

11. Guo L, Liang C, Yu T, Wang G, Li N, Sun H, et al. 3 T MRI of hepatocellular carcinomas in patients with cirrhosis: does T2-weighted imaging provide added value? Clin Radiol. 2012;67:319-28.

12. Wu LM, Xu JR, Lu Q, Hua J, Chen J, Hu J. A pooled analysis of diffusionweighted imaging in the diagnosis of hepatocellular carcinoma in chronic liver diseases. J Gastroenterol Hepatol. 2013;28:227-34.

13. Wu LM, Xu JR, Gu HY, Hua J, Chen J, Zhu J, et al. Is liver-specific gadoxetic acid-enhanced magnetic resonance imaging a reliable tool for detection of hepatocellular carcinoma in patients with chronic liver disease? Dig Dis Sci. 2013;58:3313-25.

14. Hussain HK, Barr DC, Wald C. Imaging techniques for the diagnosis of hepatocellular carcinoma and the evaluation of response to treatment. Semin Liver Dis. 2014;34:398-414. 